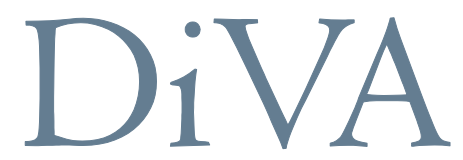

http://uu.diva-portal.org

This is an author-produced version of a paper published in Solar Energy Materials and Solar Cells. This paper has been peer-reviewed but does not include the final publisher proof-corrections or journal pagination.

Citation for the published paper:

Zhu, D., Zhao, S.

"Chromaticity and Optical Properties of Colored and Black Solar-Thermal Absorbing Coatings"

Solar Energy Materials and Solar Cells, 2010, 94(10), pp. 1630-1635

URL: http://dx.doi.org/10.1016/j.solmat.2010.05.019

Access to the published version may require subscription. 


\title{
Chromaticity and Optical Properties of Colored and Black Solar-Thermal Absorbing Coatings
}

\author{
Dechun Zhu \\ Dept. of Chemical and Materials Engineering, Hefei University, 230022, Hefei, PR. China \\ Shuxi Zhao* \\ Dept. of Engineering Sciences, Uppsala University, Box 534, SE-751 21, Uppsala, Sweden \\ E-mail:shuxi.zhao@angstrom.uu.se
}

\begin{abstract}
Two important trends in solar thermal utilization in buildings are building integration and color appearance. Coatings based on titanium alloy nitride show variety of different colors. These can be controlled by film thickness for a single layer stack, or by putting another layer on top of the first layer. Strong absorption around $500 \mathrm{~nm}$ is important to cause color appearance. Chromaticity and optical properties of colored coatings were studied and compared to black coatings. In the three-point gamut system, such colors show an additive mixture property. Solar absorptance of 0.80 to 0.95 can be obtained with thermal emittance of 0.04 to $0.09\left(100^{\circ} \mathrm{C}\right)$. These coatings provide a choice for solar thermal integration in buildings where appearance is an important feature.
\end{abstract}

Key words: Colored solar collectors; chromaticity; titanium alloy nitride; green buildings.

\section{Introduction}

Energy demand is a key issue for the economy development in every country. Energy consumption of houses and buildings contributes $40 \%$ to the total energy consumption in the European Union [1]. In China, the percentage in building consumption is 25 to 30 . Solar-thermal applications in buildings can play more important roles if two problems can be overcome: integration of solar collector installation into buildings and the color appearance

\footnotetext{
* Cooresponding author
} 
of the absorbers. For instance, solar domestic hot water systems used in China are mainly based on evacuated-tube solar collectors. Most are installed by individual families separately. A large number of such collectors have been installed in the mainland of China. Even though this probably accounts for $60 \%$ of the global solar collector area [2], the contribution to the total energy consumption is still very small. The evacuated tube configuration is not readily compatible to buildings and this limits the effective collector area in building constructions. Therefore, solar thermal collectors must be integrated into buildings, which should be considered in the design stage, not installed later. Thus, a nice appearance of the solar collectors in the construction of buildings is very important for architects and inhabitants attractive. Integration and colored absorbers will lead to a wider acceptance among building architects and users [3].

Most solar-thermal collectors in the current market are black or dark blue due to pursuing high solar absorptance (low reflectance in the eye-sensitive, i.e. visible wavelength range $(380-780 \mathrm{~nm}))$. Unfortunately, a dark color limits the architectural integration. A study shows that more than $85 \%$ of architects prefer solar collectors in building having other colors than black, even if they loose some efficiency [4]. Different ideas have been suggested, such as spectrally selective colored paint (TISS paint) [5], and colored collector glazing [6]. This paper reports on a new colored spectrally selective coating prepared by the dc magnetron sputtering technique. It consists of one or two thin layers of titanium alloy nitride (or nitro-oxide) on high infrared reflecting metal (aluminum for example). The structure of the film was identified by X-ray diffraction. Chromaticity and optical properties of the colored coatings were studied and compared to a nickel-nickel oxide composite absorber in different layer stack configurations. The way of color control is discussed, and the loss of solar absorptance is also compared to the black absorber.

\section{Samples}


The colored samples were prepared in a reactive dc magnetron sputtering unit Balzer UTT400 which is described in detail elsewhere [7]. Pure titanium (99.995\%) and aluminum (99.99\%, both are from plasma materials) were used for co-sputtering $\mathrm{Ti}_{\mathrm{x}} \mathrm{Al}_{\mathrm{y}} \mathrm{N}$ samples. The ratio of $\mathrm{Ti} / \mathrm{Al}$ was controlled by the sputtering power. Samples were named by TiAlN or TiAlON without giving a detailed chemical stoichoimetric number for simplification in this paper. The substrates were Corning glass (7059) or aluminum sheets of size $50 \mathrm{~mm} * 50 \mathrm{~mm}$. The substrates were cleaned in an ultrasonic bath, followed by de-ionized water rinsing and nitrogen gas drying. Deposition was carried out under certain sputtering conditions.

Argon flow (99.999\% pure) and nitrogen flow (99.999\% pure) were fixed at 60 and $10 \mathrm{ml} / \mathrm{min}$ respectively for nitride film deposition. Oxygen $(99.99 \%)$ flow was varied in a range from 0.15 to $2.0 \mathrm{ml} / \mathrm{min}$ under the fixed argon and nitrogen flows shown above for nitro-oxide film deposition.

For comparison, some of the previously prepared nickel-nickel oxide samples were used, including samples of single layer, double layers, and a double-layer of nickel-nickel oxide plus an anti-reflection layer. A list of the studied samples is attached in table 1.

\section{Chromaticity}

In the CIE (International Commission on Illumination) color system, colors are numerically specified in a plane described by a set of tristimulus values called $x, y$, and $z$, on the chromaticity diagram. This system offers high precision in color measurements. The parameters are based on the spectral power distribution of the light emitted from a colored object and are factored by sensitivity curves. These curves have been measured for the human eye. All existing colors can then be represented in such CIE chromaticity diagram. The tristimulus values depend on the observer's field of the view due to the nature of the distribution of cones in the eye. A set of three color-matching functions, $x(\lambda), y(\lambda)$ and $z(\lambda)$ provide the numerical 
description of the chromatic response of the observer described above. The color matching functions thus reflect the color sensitivity of the human eye. Tristimulus values $X, Y$ and $Z$ are calculated from three similar integrations of the measured or simulated spectral power distribution data $P(\lambda)$ :

$$
\begin{aligned}
& X=\int P(\lambda) x(\lambda) d \lambda \\
& Y=\int P(\lambda) y(\lambda) d \lambda \\
& Z=\int P(\lambda) z(\lambda) d \lambda
\end{aligned}
$$

The spectral power distribution $P(\lambda)$ can be the product of a source spectrum and a measured or simulated reflectance spectrum of a sample. In the case of a neutral light source,

$$
P(\lambda)=R(\lambda)
$$

where $R(\lambda)$ is the measured reflectance spectrum.

From the tristimulus values, the color coordinates $x, y, z$ are defined as

$$
\begin{aligned}
& x=X /(X+Y+Z), \\
& y=Y /(X+Y+Z), \\
& z=1-(x+y)
\end{aligned}
$$

The colors can be matched by combining a given set of three primary colors, such as red, blue, and green. Then any color can be represented in the three-point gamut in the chromaticity diagram

In this paper, only the case of a neutral light source is discussed for simplicity. Data of three color-matching functions are from Table 1 and 3 [8].

\section{Characterization}

\subsection{Optical characterization}


Solar absorptance and thermal emittance are the two basic parameters for characterization of the optical performance of a spectrally selective surface of a solar thermal absorber. They are defined as

$$
\begin{gathered}
\alpha_{S}=\frac{\int_{0.3}^{4.0}(1-R(\lambda)) I_{S}(\lambda) d \lambda}{\int_{0.3}^{4.0} I_{S}(\lambda) d \lambda} \\
\varepsilon_{t}=\frac{\int_{2.0}^{100}(1-R(\lambda)) I_{b}(\lambda, T) d \lambda}{\int_{2.0}^{100} I_{b}(\lambda, T) d \lambda}
\end{gathered}
$$

where, $R(\lambda)$ is the measured reflectance at a specific wavelength $\lambda, I_{s}(\lambda)$ is the solar spectral radiation and $I_{b}(\lambda, T)$ is the blackbody spectral radiation. $\mathrm{T}=373 \mathrm{~K}$ is used for low temperature solar thermal applications.

Reflectance was measured by a Perkin-Elmer Lamda 900 UV/VIS/NIR double beam spectrophotometer in the wavelength range 0.3 to $2.5 \mu \mathrm{m}$. It was equipped with an integrating sphere to allow for measurements of rough substrate surfaces [9]. In the infrared range $(2.5$ to $22 \mu \mathrm{m})$, a Fourier Transform Infrared Reflectance (FTIR) spectrometer TENSOR 27 (Bruker Optics) was used. It was equipped with an integrating sphere coated with gold. These data were then used to calculate solar absorptance and thermal emittance of studied coatings.

\subsection{Structure characterization}

X-ray diffraction (XRD) was used to determine the crystalline phase and structural properties. XRD measurements were performed by Siemens D5000 diffractometer operating with a grazing incidence angle of one degree in parallel beam geometry using $\mathrm{CuK}_{a}$ radiation (this wavelength is $1.54 \AA$ ). The diffraction pattern was analyzed between $20^{\circ}$ and $90^{\circ}$. 


\section{Results and discussion}

\subsection{Optical properties of single layer absorbing coatings}

From the energy conversion efficiency point of view, solar absorbers need as low reflectance as possible in the solar spectral range to maximize the capture of solar energy. Meanwhile the reflectance in the infrared wavelength range should be as high as possible to minimize radiation losses [10]. Many different structures/materials have been proposed to reach this goal. One popular type of coating material on the market is the metal-dielectric composite. Metal-dielectric composites used for solar-thermal energy conversion consist of very fine metal particles embedded in a dielectric host, also known as granular or cermet (ceramic metal) materials [11]. Such composites are strongly absorbing in the solar spectral range. In the infrared spectral range, they rapidly become highly transparent with increasing wavelength. When such a composite is coated on a highly reflecting metal surface as a thin film, the resulting absorber-reflector tandem shows excellent spectrally selective character. Such a composite is usually produced with a compositional gradient so that the metallic content is gradually reduced towards the surface in order to reduce front surface reflection losses. A three-layer configuration (base-layer + middle layer + anti-reflection or AR layer) is one simple type of gradient coating in production [12]. The base layer is the most important layer for solar absorptance in three-layer configurations. Therefore a single-layer of a composite coating was used as reference for colored single-layer coatings, which provided a simple way to study the mechanisms causing color.

Figure 1 shows the reflectance spectrum of one selected sample " $B-1 L$ ", which is a homogeneous single layer coating using a nickel-nickel oxide composite $(150 \mathrm{~nm})$. Such a coating was carefully studied earlier since it was used as the base-layer in three-layer absorbers. For single homogeneous layers of ceramic-metal coatings, only one interference minimum is observed if there is no other absorption mechanism in 
the solar wavelength range such as band gap absorption. This interference minimum of the single layer thin film is located at a wavelength around 1.4 to $2 \mu \mathrm{m}$ for a film thickness of $150 \mathrm{~nm}$ to $200 \mathrm{~nm}$, as shown in figure 1 . Such a surface usually appears black or dark-blue due to its low reflectance in the visible wavelength range.

\section{Figure 1}

Figure 2 shows the reflectance spectrum of single titanium aluminum nitride TiAlN " $C-1 L R$ " on an aluminum substrate. A significant difference is the separation into two peaks in the visible wavelength range. The reflectance minimum at a wavelength around $500 \mathrm{~nm}$ is not due to interference, which suggests absorption by the band structure of sputtered titanium aluminum nitride. A consequence is that the two peaks appear in the visible wavelength range. The reflectance in the solar wavelength range is low, thus the solar absorptance of the TiAlN thin film is higher than for the single layer stack, which was 0.81 for the typical single $\mathrm{Ni}-\mathrm{NiO}$ thin film coating in figure 1 . The absorption at $500 \mathrm{~nm}$ enhances the solar absorptance to be 0.86 . This absorption is also important for the color appearance. Sample " $C$ - $1 L R$ " in figure 2 appears red due to the peak at $683 \mathrm{~nm}$.

\section{Figure 2}

\subsection{Thickness effect}

Our results show that the peaks can be shifted by varying the film thickness. Figure 3 and 4 (together with figure 2) show the reflectance spectrum of single TiAlN layer of different film thickness where the relative thickness was determined from the deposition time. Figure 3 shows the reflectance spectrum of the thinnest film " $C-1 L Y$ " among the three which was deposited for 8 minutes (the thinnest among three). The corresponding thin film appears yellow (peak at $575 \mathrm{~nm}$ ). Figure 4 shows the thickest film " $C-1 L B$ " deposited for 11 minutes with a blue color (peak at $463 \mathrm{~nm}$ ). The middle thickness among three samples " $C-1 L R$ " is shown in figure 2 deposited for 9 
minutes (peak at $683 \mathrm{~nm}$ ). It shows how the relative intensity of the peaks affects the color of the samples.

\section{Figure 3}

\section{Figure 4}

\subsection{Chromaticity diagram of single-layer absorber}

Figure 5 shows the color coordinates of the three samples in the CIE tristimulus chromaticity diagram. The calculation was carried out using a neutral light source according to the discussion in section 3 . If the reflectance in the whole calculated wavelength range has equal value, the color refers to be neutral and is at the location with $x=y=z=0.333$, marked as "Neutral" in figure 5. The color at that location is white, black, or grey. In reality, the location of a neutral color does not need to be exactly at that one point. There is an area around the "neutral location" corresponding to a neutral (or black) color [13]. The location of sample " $B-1 L$ " is very close to "Neutral" due to its "mono-reflectance" behavior in the visible wavelength range shown in figure 1.

The color locations from figure 2 to 4 are marked by " $C-1 L R$ ", " $C-1 L Y$ ", and " $C-1 L B$ " respectively. As can be seen, the locations are well outside the neutral area. The color called "Red", "Blue"... above is according to the feeling from our eye. The numerical location in the diagram is the precise description of the color. In the three-point gamut in the chromaticity diagram [14], the color of the single layer with two peaks in figure 2 shows the property of an additive mixture. To find out the effect of the main peak in the visible wavelength range, we calculated the color locus removing the other peak. These "dominating" loci are also displayed in figure 5 marked by " $R_{\text {peak }}$ ", " $Y_{\text {peak }}$ ", " $B_{\text {peak }}$ ". The locus difference between " $C-I L R$ " and " $R_{\text {peak }}$ " is big indicating the additive mixture property of the color due to the two peaks shown in figure 2 . The same situation appears in " $C-1 L B$ " color shown in figure 4. However, the locus 
difference between " $C-1 L Y$ " and " $Y_{\text {peak" }}$ is small. The possible reason is that the intensity of the main peak dominates the visible wavelength range in figure 3.

\section{Figure 5}

The discussion in this section shows the effect of strong absorption on color vision. The color will be close to neutral for absorbers without such absorption. The reason for the colors of the studied samples may be due to the band structure of transition metal nitride.

\subsection{Two and three-layer samples}

For solar-thermal energy conversion systems it would be desirable to capture all incident light to the absorbing surface and then deliver a high possible fraction of the captured energy to the user through a working fluid. This principle is the same for the colored absorbers. For the composite single layer shown in figure 1, the low solar absorptance is mainly due to a comparatively high refractive index of composite nickel-nickel oxide which causes a significant surface reflectance loss. If another layer with lower refractive index material is coated on top of the sample in figure 1, the solar absorptance will be increased. Figure 6 shows the result. Sample " $B-2 L$ " contains two thin film layers: a $50 \mathrm{~nm}$ nickel-nickel oxide thin film with low metallic content coated on top of the $150 \mathrm{~nm}$ nickel-nickel oxide thin film with higher metallic content as base-layer (similar to sample shown in figure 1). The spectrum has two interference minima: minimum at $0.7 \mu \mathrm{m}$ is due to an interference minimum of the top layer. The reflectance in the solar range is lower than that shown in figure 1 due to a lower refractive index of the top layer [15]. As a consequence, the solar absorptance increases from 0.81 to 0.91 .

\section{Figure 6}


It works in a similar way for a colored single layer coating. Figure 7 shows the spectrum " $C-2 L G$ " after depositing a thin layer on top of the base layer: a layer of TiAlON (reacted with oxygen to reduce refractive index) on top of the TiAlN layer from figure 2. The solar absorptance increases from 0.86 to 0.92 by the two-layer stack. The difference between " $B-2 L$ " and " $C-2 L G$ " is that there are three minima in the solar wavelength range in the spectrum of sample " $C-2 L G$ ": two are caused by interference; one is due to the material absorption. This resulted in a dominating peak in the visible range at $515 \mathrm{~nm}$ which gives a strong color appearance.

\section{Figure 7}

If an anti-reflection (AR) layer is further deposited on top of the above graded two layers (shown in figure 7), the solar absorptance can be further enhanced to 0.95 . Figure 8 shows the reflectance spectrum of sample " $C-3 L$ ". The figure shows that the material absorption feature remains, resulting in a peak at $615 \mathrm{~nm}$. This peak gives the color appearance.

\section{Figure 8}

Figure 9 shows the loci of the two and three-layer colored samples in the chromaticity diagram (" $C-2 L G$ ", and " $C-3 L ")$. The location of one-layer and two-layer nickel-nickel oxide composite samples are also displayed in figure 9. It also shows that the two black samples have different location according to the reflectance change in the visible range. The locations are both in an area close to the neutral point. For the colored samples, the color coordinates are out of the neutral area.

\section{Figure 9}

One should note that the locus in the chromaticity diagram is calculated assuming a neutral light source. In daylight, using the sun as the light source, the color 
appearance would be slightly modified, but with no change of color. Red would still be red only with slightly different $x, y$-values.

\subsection{Structure characterization}

The XRD pattern of single-layer sample " $C-1 L R$ " in figure 10 shows the TiAlN structure with preferred orientation (111) [16].

\section{Figure 10}

\subsection{Loss of solar absorptance}

The solar absorptance of most black absorbers for low temperature applications in the market is about $95 \%$ as an average value. For colored samples, the loss of solar absorptance was calculated with respect to the value of $95 \%$. The results are listed in the attached table 2 . About $10 \%$ to $15 \%$ of solar absorptance is lost for single-layer colored coatings. But the loss is reduced to only $3 \%$ for double-layer colored coatings. The loss can be neglected if an AR coating is added. Therefore, the loss can be reduced to a small value for such colored coatings, and the compromise between solar absorptance and color appearance should be accepted by the users.

\subsection{Potential application}

Embedding solar collectors into roofs of buildings is one popular integration design during recent years. Colored solar-thermal coatings are well suited for this application. There is already an existing market selling metallic roofs with different colors called "Colored Steel Tile". Red and blue are the most popular colors for such roofs used in some area in China (like north-east China, east coast city etc.). Originally, they were designed for preventing rainwater leaks. If spectrally selective colored coatings are used as roof material, a rather large absorbing area is available. The collected energy will contribute to domestic hot water, space heating, and even as an energy source for cooling. The potential market is huge, but it needs other devices to form a complete 
set from an engineering point of view, such as thermal energy transfer systems, heat storage, and heat exchange devices.

\section{Conclusions}

TiAlN is a promising spectrally selective absorber material. Typical solar absorptance values of such a single layer coating are around 0.8 to 0.86 with a thermal emittance of 0.04 to 0.08 . Absorption around $500 \mathrm{~nm}$ of the single layer coatings forms two peaks in the visible wavelength range which results in color appearance. Color of single layer coatings has been shown to be a function of film thickness. For a two-layer stack of TiAlON/TiAlN on an aluminum substrate, three peaks in the visible range are formed. As a result, higher solar absorptance can be obtained with maintained color appearance. A solar absorptance of 0.92 and an emittance of 0.08 were obtained for our green colored sample " $C-2 L G$ ". If an anti-reflection layer is added on top, the solar absorptance is close to the value of the black solar absorbers commonly found on the market today. The locus in the chromaticity diagram shows the additive mixture property of color from the studied samples. A compromise between maximum solar absorptance and color appearance is needed for the application of building integration. Such colored coatings are suitable candidates for energy-efficient buildings, for example as integrated colored roofs, or facades with spectrally selective properties.

\section{Acknowledgement}

The authors would like to thank Professor Arne Roos for his assistance in the optical characterization and for kindly helping to correct the English.

Thanks to the Faculty of Science and Technology, Uppsala University for the financial support for the international cooperation between Hefei and Uppsala University.

Thanks to the Natural Science Foundation of Anhui Educational Commission (KJ2009B036) for the work. 


\section{Reference}

1. A.G. Hestnes, Building integration of solar energy system, Solar Energy 67 (1999) 181-187.

2. R. Wang, X. Zhai, Development of solar thermal technologies in China, Energy, In Press (2009).

3. M. Probst, C. Roecker, Towards an improved architectural quality of building integrated solar thermal system (BIST), Solar Energy, 81 (2007) 1104-1116.

4. A. Schuler, C. Roecker, J-L. Scartezzini, J. Boudaden, I.R. Videnovic, R.S-C. Ho, P. Oelhafen, On the feasibility of colored glazed thermal solar collectors based on thin film interference filters, Solar Energy Materials and Solar Cells, 84 (2004) 241-254.

5. B. Orel, H. Spreizer, A. Surca Vuk, M. Fir, D. Merlini, M. Vodlan, and M. Kohl, Selective paint coatings for colored solar absorbers: Polyurethane thickness insensitive spectrally selective (TISS) paints (Part II), Solar Energy Materials and Solar cells, 91 (2007) 108-119.

6. J. Boudaden, R. Ho, P. Oelhafen, A. Schuler, C. Roecker, J. Scartezzini, Towards colored glazed thermal solar collectors, Solar Energy Materials and Solar cells, 84 (2004) 225-239.

7. S. Zhao, "Spectrally selective solar absorbing coatings prepared by dc magnetron sputtering", PhD. Thesis (Uppsala University, Sweden, 2007, ISSN 1651-6214) 36-37.

8. G. Wyszecki, Colorimetry, in: Walter G. Driscoll (Ed.) Handbook of Optics, Optical Society of American, McGraw-Hill Book Company, New York, P9-6 to $\mathrm{P} 9-12$.

9. A. Roos, Use of an integrating sphere in solar energy research, Solar Energy Materials and Solar cells, 30 (1993) 77-94.

10. Agnihotri and B.K. Gipta, Solar Selective Surfaces, A Wiley-Interscience publication, 1981, New York, pp88. 
11. R.A. Buhrman and H.G. Craighead, Composite Film Selective-Absorbers, in Lawrence E. Murr (Ed.) Solar Materials Science, 1980, Academic Press, New York, pp277-292.

12. S. Zhao, E. Wäckelgård, Optimization of solar absorbing three-layer coatings, Solar Energy Materials and Solar Cells, 90 (2006) 243-261.

13. http://hyperphysics.phy-astr.gsu.edu/hbase/vision/cie.html

14. http://cs.haifa.ac.il/hagit/courses/ist/Lectures/Demos/ColorApplet2/a_chroma. $\underline{\mathrm{html}}$

15. Q. Zhang, D.R. Mills, New cermet film structures with much improved selectivity for solar thermal applications, Applied Physics Letters, 60 (1992) 545-547.

16. C. Hsu, M. Chen, K. Lai, Corrosion resistance of TiN/TiAlN-coated ADI by cathodic arc deposition, Materials Science and Engineering A, 421 (2006) 182-190. 


\section{Tables}

Table 1: list of samples

\begin{tabular}{|l|c|c|l|l|l|}
\hline New Lable & $\alpha$ & $\varepsilon$ & No. Layer & Coating materials & Color \\
\hline$B-1 L$ & 0.81 & 0.02 & 1 layer & Ni/NiO & Black \\
\hline$C-1 L R$ & 0.86 & 0.04 & 1 layer & TiAlN & Red \\
\hline$C-1 L Y$ & 0.81 & 0.06 & 1 layer & TiAlN & Yellow \\
\hline$C-1 L B$ & 0.86 & 0.08 & 1 layer & TiAlN & Blue \\
\hline$B-2 L$ & 0.91 & 0.06 & 2 layers & Ni/NiO & Black \\
\hline$C-2 L G$ & 0.92 & 0.08 & 2 layers & TiAlN+TiAlON & Green \\
\hline$C-3 L$ & 0.95 & 0.09 & 3 layers & TiAlN+TiAlON+AR & Dark red \\
\hline
\end{tabular}

Table 2. Loss of solar absorptance compared to black sample $(\alpha 1=0.95)$

\begin{tabular}{|c|c|c|c|c|c|c|}
\hline & $\boldsymbol{C}-1 \boldsymbol{L Y}$ & $\boldsymbol{C - 1 L R}$ & $\boldsymbol{C}-1 \boldsymbol{L B}$ & $\boldsymbol{C}-2 \boldsymbol{L G}$ & $\boldsymbol{C}-3 \boldsymbol{L}$ & $\boldsymbol{R e f}$. \\
\hline$\alpha$ & 0.81 & 0.86 & 0.86 & 0.92 & 0.95 & $\alpha 1=0.95$ \\
\hline$\Delta \alpha(\alpha-\alpha 1)$ & -0.14 & -0.09 & -0.09 & -0.03 & 0 & \\
\hline$\Delta \alpha / \alpha 1$ & -0.15 & -0.09 & -0.09 & -0.03 & 0 & \\
\hline
\end{tabular}




\section{Figure Captions}

Figure 1. Reflectance spectrum of single-layer sample " $B-l L$ " of Ni-NiO composite on aluminum substrate. Minimum at $1.4 \mu \mathrm{m}$ is due to thin film interference.

Figure 2. Reflectance spectrum of single-layer sample " $C-1 L R$ " of TiAlN on aluminum substrate. Absorption at $500 \mathrm{~nm}$ is due to the property of coating materials. Red color appears due to the peak of $683 \mathrm{~nm}$.

Figure 3. Reflectance spectrum of single-layer sample " $C-1 L Y$ " of TiAlN on aluminum substrate. The thickness of film is thinner than that of " $C-1 L R$ ". Yellow color appears due to the peak of $575 \mathrm{~nm}$.

Figure 4. Reflectance spectrum of single-layer sample " $C-1 L B$ " of TiAlN on aluminum substrate. The thickness of film is thicker than that of "C-lLR". Blue color appears due to the peak of $463 \mathrm{~nm}$.

Figure 5. The chromaticity diagram of single layer samples. It shows the additive mixture of color property from colored samples.

Figure 6. Reflectance spectrum of two-layer sample " $B-2 L$ ” of Ni-NiO (low Ni)/Ni-NiO (high Ni) on aluminum substrate.

Figure 7. Reflectance spectrum of two-layer sample " $C-2 L G$ " of TiAlON/TiAlN on aluminum substrate. Green color appears due to the peak of $515 \mathrm{~nm}$.

Figure 8. Reflectance spectrum of three-layer sample " $C-3 L$ " of AR/TiAlON/TiAlN on aluminum substrate. Dark-red color appears due to the peak of $615 \mathrm{~nm}$.

Figure 9. The chromaticity diagram of two and three-layer samples. It shows the additive mixture 
of color property from colored samples.

Figure 10. The XRD of sample " $C-1 L R$ " shows the pattern of TiAlN, preferring orientation (111).

\section{Figures}

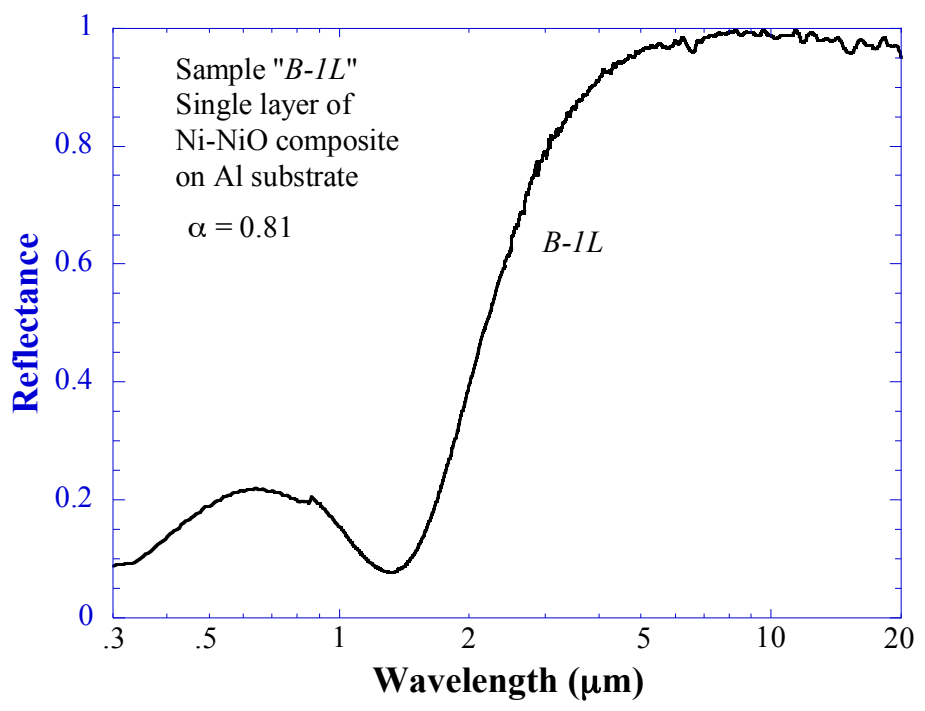

Figure 1

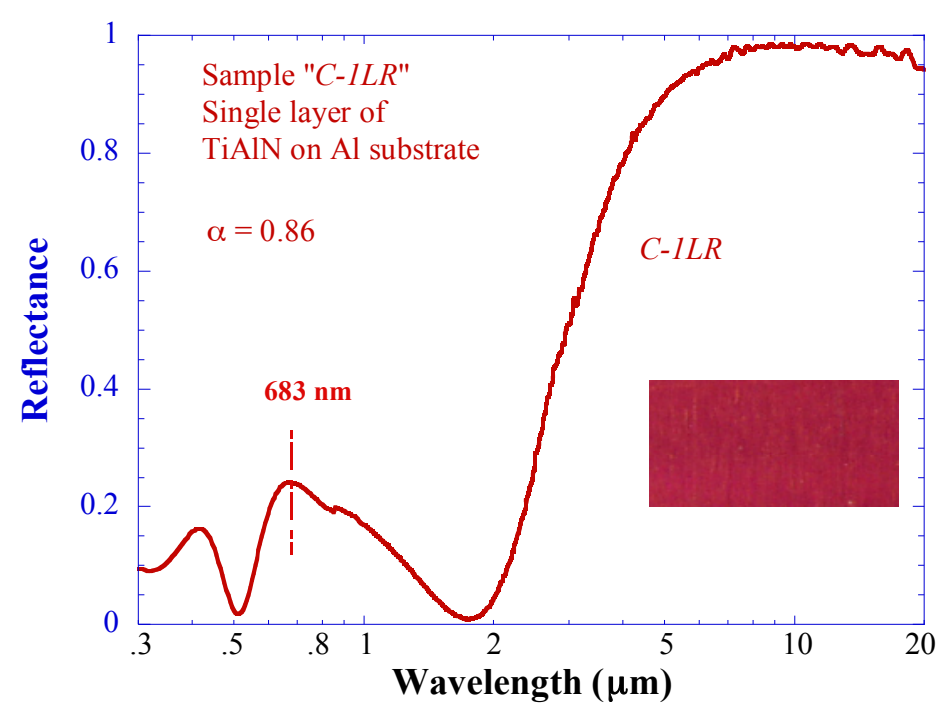

Figure 2 


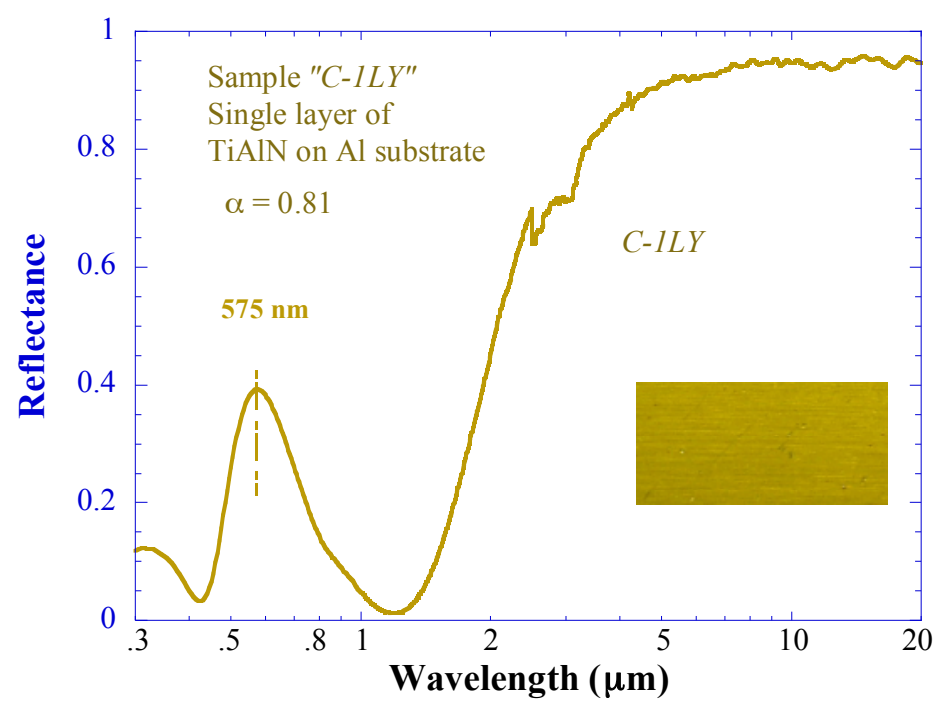

Figure 3

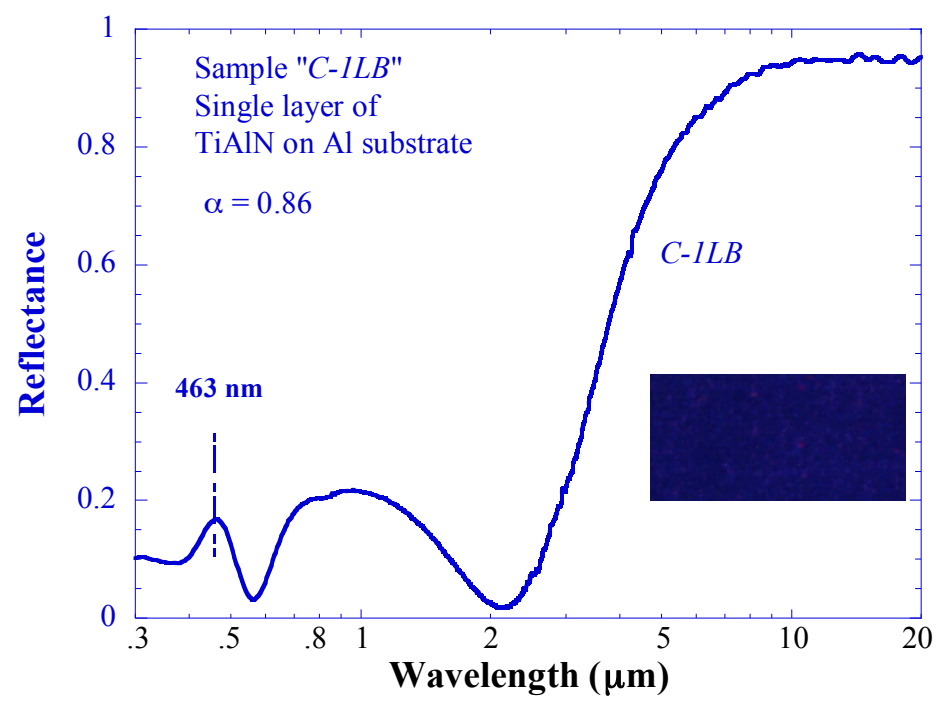

Figure 4 


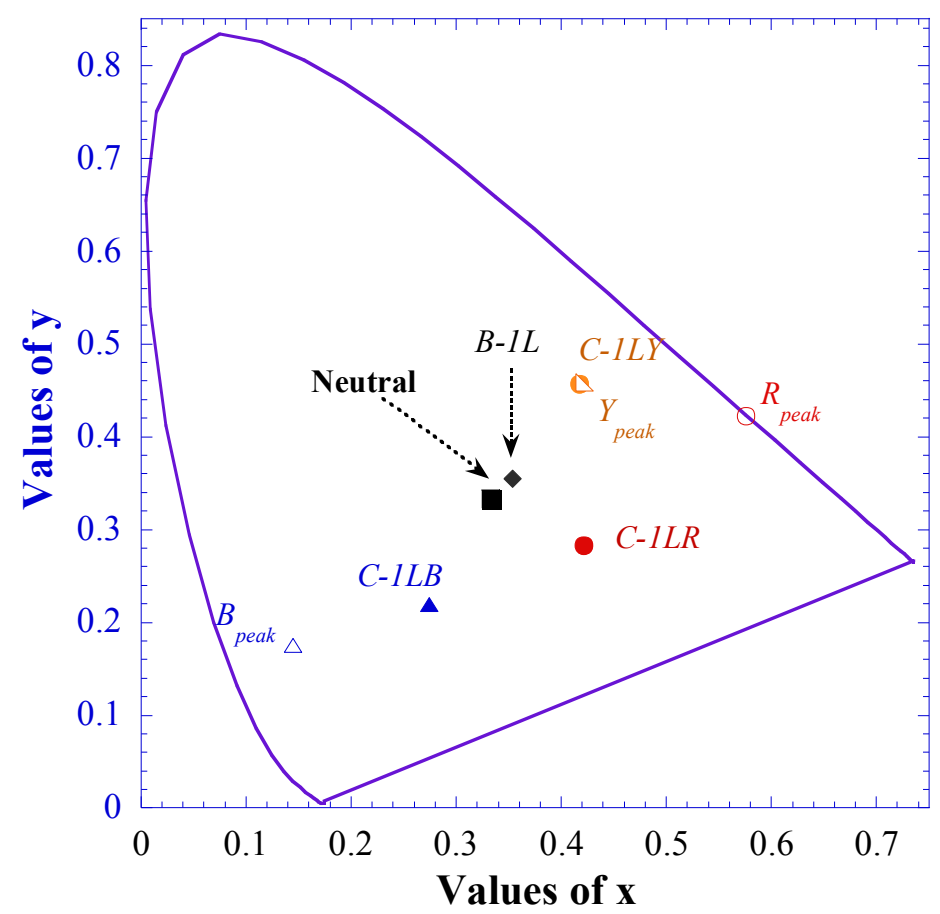

Figure 5

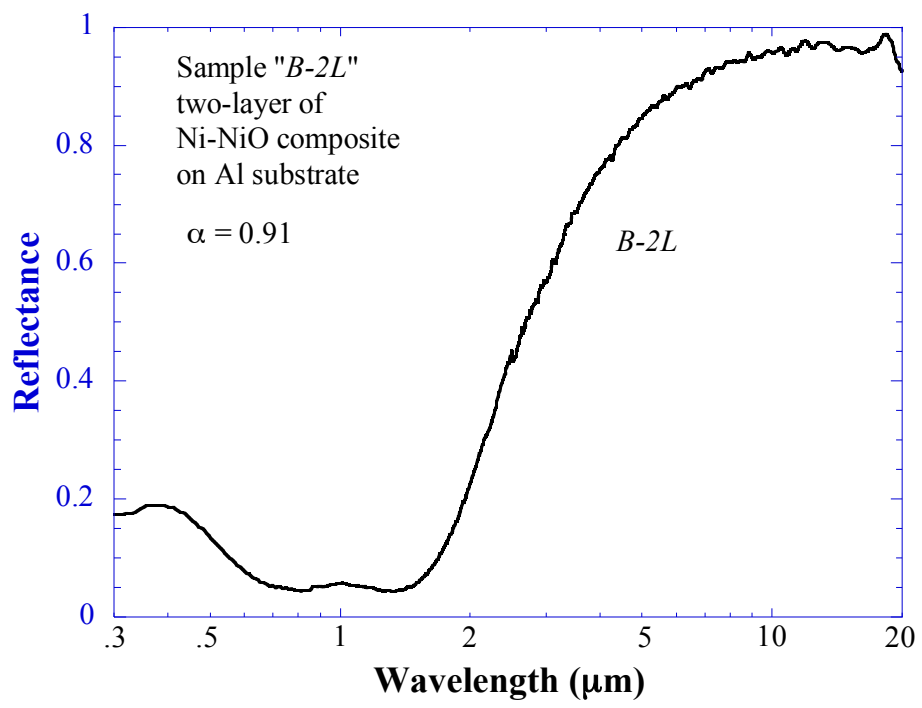

Figure 6 


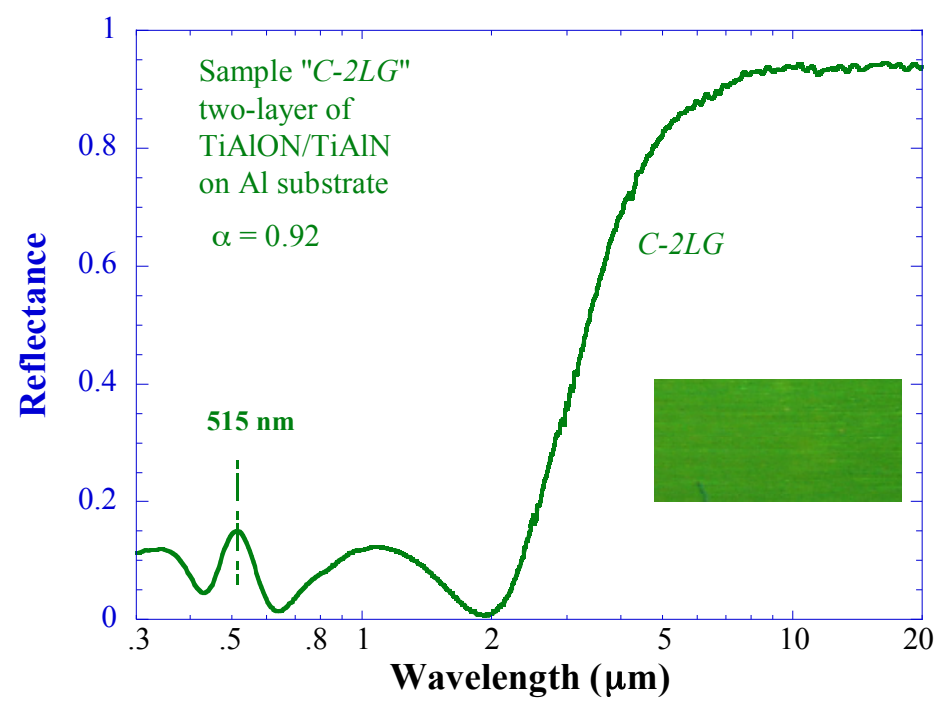

Figure 7

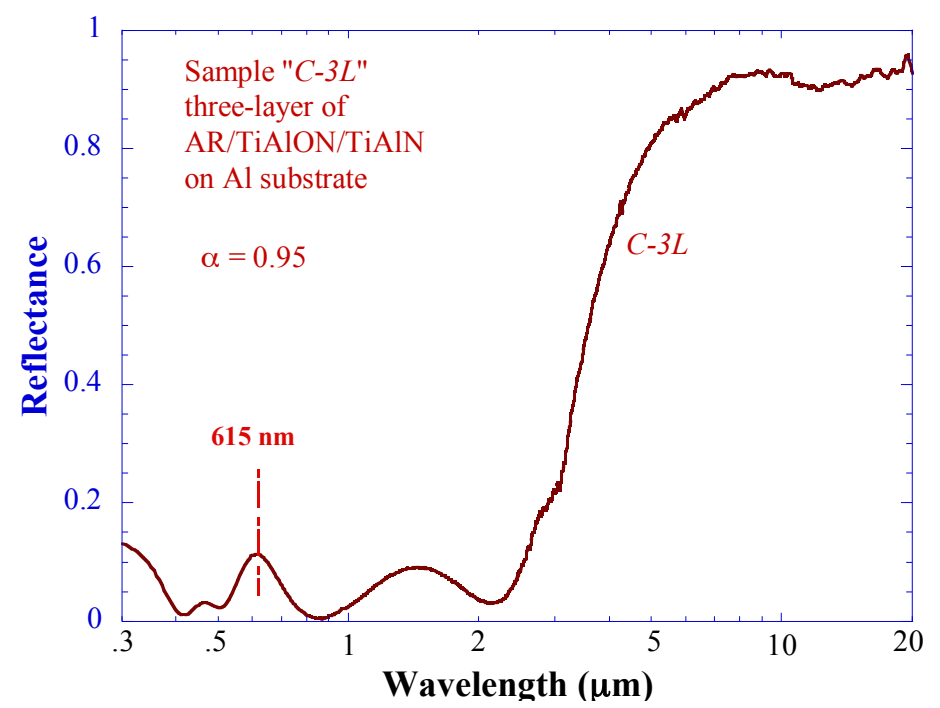

Figure 8 


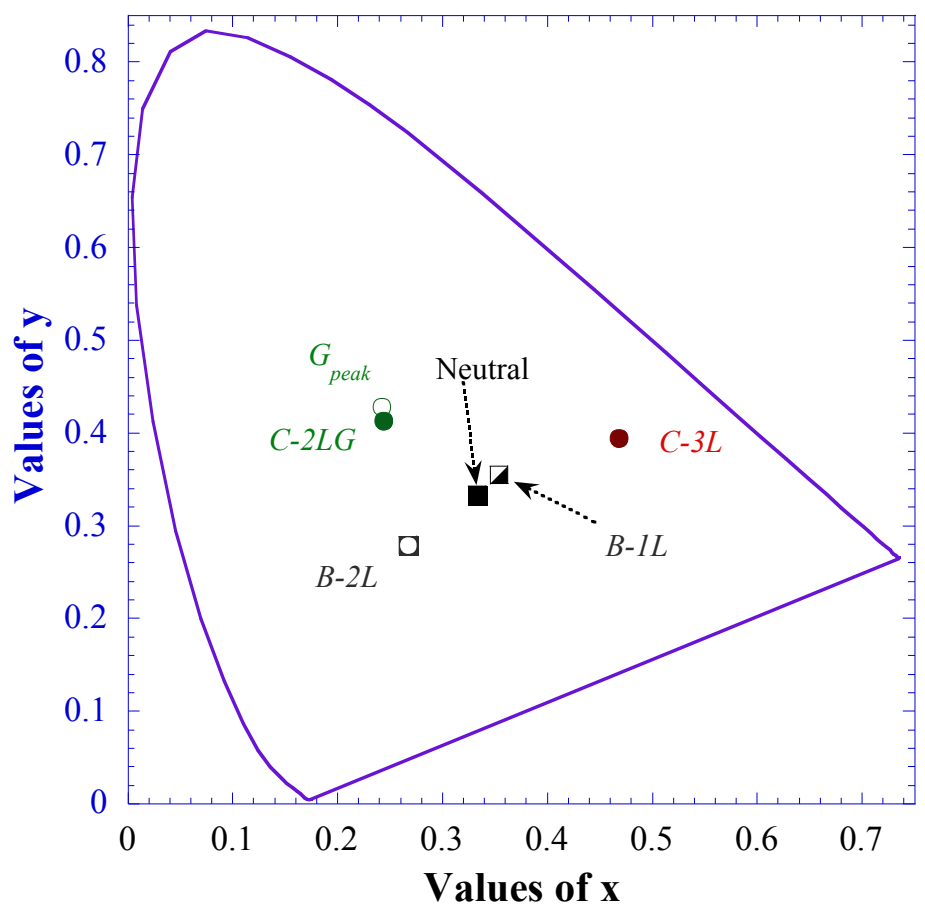

Figure 9

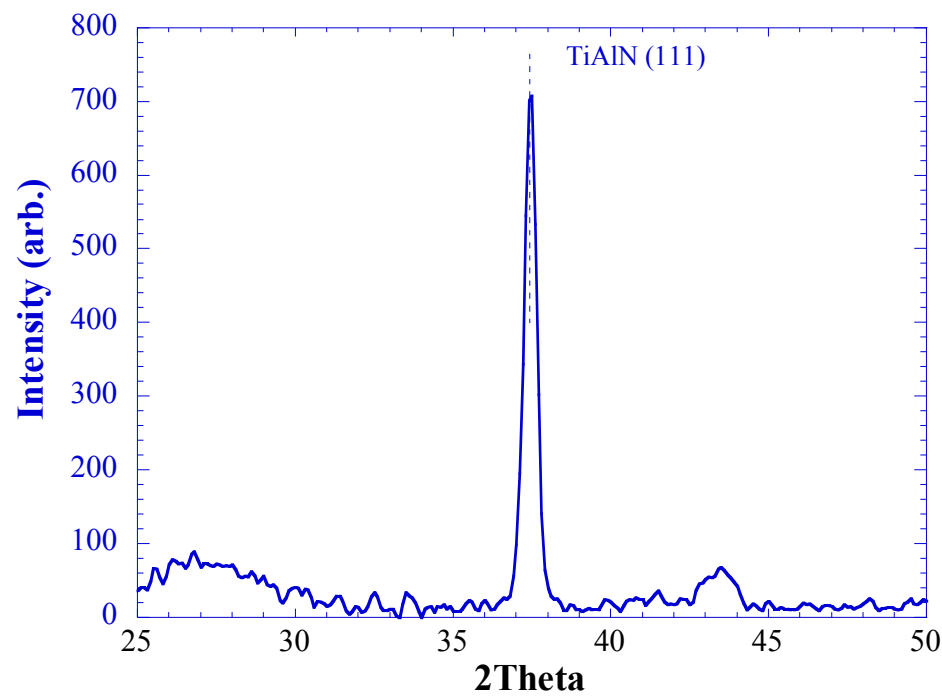

Figure 10 